\title{
VIAGGIO DI LEONARDO FEA \\ IN BIRMANIA E REGIONI VICINE
}

\section{LXXVIII.}

NOUVELLE ESPÉCE CAVERNICOLE DE LA FAMILLE DES BLATTAIRES PAR I. BOLIVAR

\section{Spelaeoblatta gen. nov.}

Statura parva. Caput oblongum, elongatum. Oculi nulli. Antennae valde elongatae. Palporum max. articulus ultimus apicem versus incrassatus, apice truncato-rotundatus. Pronotum antice valde arcuatum, postice truncatum. Elytra subcornea, haud venosa, lobiformia, subquadrata et lateralia. Metanotum angulis posticis distincle productis. Alae nullae. Femora subtus parum spinosa. Tibiae multispinosae, supra rotundatae atque biseriatim spinosae. Tarsi elongati, apice subtus haud areolati. Arolio nullo. Abdomen segmentis ultimis dorsalibus angustis, transversis. Lamina supraanalis triangularis, apice rotundata. Segmentum ventrale ultimum valvulis instructum. Cerci imperfecte articulati, apice aculeati.

Corps oblong. Tête étroite et assez allongée, cachée sous le pronotum, ne laissant apercevoir qu'une très petite partie du vertex; les yeux manquent complétement; les scrobes des antennes sont assez grandes et arrondies et le troisième article de ces organes est un peu plus long que le deuxième, conformément aux observations de feu Stål et de Mr. Brunner qui ont démontré que cet article est plus long que le deuxième chez le Périplanètides aptères ou brachyptères, relation qui subsiste pour les deux sexes d'une même espèce lorsque l'un d'eux est 
ailé l'autre étant aptère; les antennes sont du reste très longues et fines et leur premier article est grand et presque cylindrique bien qu'un peu atténué à la base et les deux suivants sont allongés et un peu plus longs pris ensemble que le premier, les suivants jusqu'au premier tiers sont courts et subglobuleux, devenant insensiblement coniques et plus longs que larges dans le reste jusqu'à l'extrémité. Tous les articles, à l'exception toutefois des premiers, sont pubescents. Mandibules fortes et brunàtres. Palpes maxillaires grêles et assez longs, avee les articles basilaires courts et globuleux, le troisième cylindrique et allongé, étant plus long que le quatrième qui est épaissi vers l'extrémité et plus long que le cinquième, celui-ci est conique et plus gros vers l'extrémité, subarrondi au bout et couvert de longs poils disposés tout autour. Palpes labiaux triarticulés, à dernier article subcylindrique et arrondi à l'apex.

Pronotum légèrement convexe, avec le bord antérieur arqué en demi cercle et le postérieur tronqué ou très légèrement sinueux au milieu, les angles latéraux sont arrondis. Mesonotum avec le bord postérieur un peu convexe. Metanotum sinué postérieurement avec les angles postérieurs saillants. Élytres très courts, latéraux, ne dépassant presque pas le bord postérieur du mesonotum, de consistance cornée et sans nervations. Ailes nulles.

Pattes longues et grêles; les hanches de la première paire offrent à l'extrémité du bord inférieur un lobe saillant obtus. Cuisses antérieures comprimées et légèrement fusiformes, plus longues que les jambes correspondantes qui à leur tour sont plus courtes que les tarses de la mème paire, cette relation change dans les pattes suivantes, les jambes intermédiaires étant aussi longues que les cuisses et les postérieures plus longues qu'elles, tandis que les tarses des intermédiaires ont presque la longueur des tibias correspondants et ceux des postérieurs sont plus courts que les jambes. Toutes les cuisses sont épineuses inférieurement, mais les épines sont très peu nombreuses, elles ont en outre des poils épars et du cóté dorsal elles sont armées d'une épine apicale. Les jambes portent deux séries d'épines 
du côté externe qui est arrondi et se terminent par de longs éperons, les jambes postérieures sont visiblement arquées. Les tarses sont filiformes et le premier article est aussi long dans les pattes intermédiaires et plus long dans les autres que les autres articles du tarse réunis. L'arolium manque de même que les aires apicales des articles.

Abdomen ovale avec le bord postérieur des premiers arceaux dorsaux un peu sinueux au milieu. Les derniers arceaux sont très courts et fortement transverses. La plaque suranale est grande, en triangle arrondi et entière. Dernier segment ventral très large à la base mais fortement rétréci jusqu'à l'insertion des valves, celles-ci plus longues que larges. Cerques longs' et forts, aplatis, à premier article grand et conique, le reste imparfaitement articulé est terminé par une longue et forte épine.

C'est en étudiant au microscope les jambes de cette petite espèce que je me suis rendu compte de la disposition terminale de ces organes, disposition qui est essentiellement la même que celle que l'on trouve chez d'autres espèces, mais qui peut offrir des variations qui méritent peut être d'être suivies avec méthode car il est probable qu'elles puissent être d'interêt pour la caractérisation de certains groupes.

De même que le genre Nocticola mihi qui ne renferme que deux espèces de l'île de Luzon et qui sont aussi cavernicoles, ce nouveau genre manque d'arolium aux tarses, caractère qui permet de ranger les deux genres l'un à côté de l'autre et les séparer du reste des Périplanètides. Cette distinction à part, ils rentreraient dans la division 1' du Système des Orthoptères de Mr. Brunner (pag. 34) " Tibiae supra biseriatim spinosae " et dans la sous division 2' par leurs jambes arrondies du côté extérieur, d'autant que je peux en juger par les exemplaires presque uniques du genre Nocticola et le seul exemplaire du Spelaeoblatta que j'ai pu examiner. La nouvelle coupe taxonomique qu'il faudrait ajouter au tableau des Periplanetidae pourrait encore se caractériser par le manque des yeux ou la disposition très simple qui en offrent ces organes dans les autres (Nocticola Simoni Bol.), ainsi que par le racourcissement des élytres, 
le manque d'ailes et la disposition particulière des cerci; ces organes offrent comme je l'ai dit ailleurs (Ann. de la Soc. ent. de France 1892, p. 33) une grande facilité à se détacher restant en place le premier article seulement, le reste étant imparfaitement articulé bien que les articulations soient assez visibles dans quelques cas (Spelaeoblatta).

La forme de la tête, qui est oblongue et assez allongée, celle des palpes maxillaires, du pronotum et des élytres (ces derniers manquent tout à fait dans les $\subsetneq q$ du Nocticola), ainsi que la disposition du bord postérieur du metanotum, tronqué chez les Nocticola et assez profondement sinué avec les angles postérieurs saillants dans le Spelaeoblatta et finalement la forme des pièces

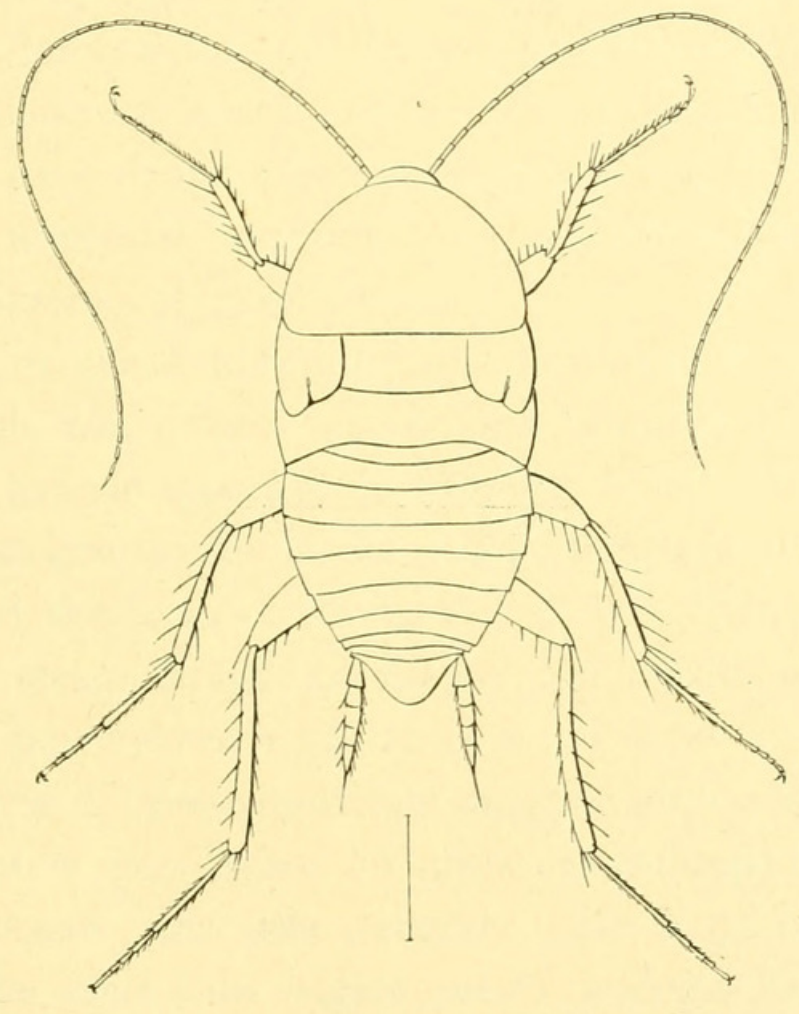

Spelaeoblatta Gestroi.

anales et des cerques caractérisent ce nouveau genre et justifient sa formation. Il ne renferme qu'une seule espèce qui habite dans les cavernes de Birmanie et que je me fais un devoir de dédier au savant sous-directeur du Musée Civique de Gênes Mr. le Dr. Gestro à qui je dois sa connaissance. 
Spelaeoblatta Gestroi sp. n.

Colore pallido. Elytra lobiformia, lateralia, subquadrata, extus distincte rotundata, postice oblique truncata el subsinuata, pone medium metanoti haud extensa. Lamina supraanalis transversa, trigona, postice rotundata, integra. Valvulis geni'alibus longioribus quam latioribus. Pedibus testaceis $९$.

Long. corporis $9^{\mathrm{mm}}, \breve{5}$.

Loc. Caverne de Jadò, Carin Asciuii Chebà, 1200-1300 m. Janvier, 1888. Provenant du voyage de Mr. L. Fea. 


\section{$2 \mathrm{BHL}$ Biodiversity Heritage Library}

1897. "Viaggio di Leonardo Fea in Birmania e regioni vicine. LXXVIII. Nouvelle espece cavernicole de la famille des Blattaires." Annali del Museo civico di storia naturale di Genova 38, 32-36. https://doi.org/10.5962/bhl.part.8730.

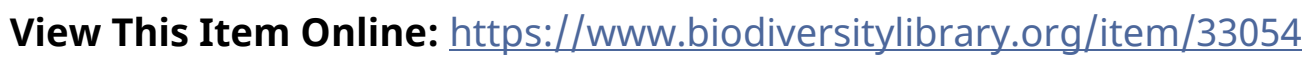

DOI: https://doi.org/10.5962/bhl.part.8730

Permalink: https://www.biodiversitylibrary.org/partpdf/8730

\section{Holding Institution}

Harvard University, Museum of Comparative Zoology, Ernst Mayr Library

\section{Sponsored by}

Harvard University, Museum of Comparative Zoology, Ernst Mayr Library

\section{Copyright \& Reuse}

Copyright Status: NOT_IN_COPYRIGHT

This document was created from content at the Biodiversity Heritage Library, the world's largest open access digital library for biodiversity literature and archives. Visit BHL at https://www.biodiversitylibrary.org. 